\title{
GESTIÓN DE RESIDUOS SÓLIDOS DOMICILIARIOS EN LA CIUDAD DE VILLAVICENCIO. UNA MIRADA DESDE LOS GRUPOS DE INTERÉS: EMPRESA, ESTADO Y COMUNIDAD.
}

\author{
Ángela María Niño Torres (iD \\ Juan Manuel Trujillo González (i) ${ }^{2}$ \\ Adriana Paola Niño Torres (I)
}

Recibido el 20 de junio de 2015, aprobado el 2 de marzo de 2016 y actualizado el 17 de diciembre de 2016

DOI: 10.17151/luaz.2017.44.11

\section{RESUMEN}

La gestión ambiental implica un manejo participativo en la solución de los problemas (García et al., 2014), al respecto el artículo presenta los resultados en cuanto a la percepción de los grupos de interés: Empresa, Estado y Comunidad, sobre aspectos relacionados con la gestión de residuos sólidos, este trabajo se enfocó en conjuntos residenciales de la ciudad de Villavicencio, con el objetivo de conocer la situación actual y establecer aspectos críticos como punto de partida para mejorar los procesos actuales en cuanto a la sostenibilidad de la gestión de residuos sólidos en la ciudad. Según el enfoque metodológico Investigación-Acción y la normatividad vigente, que consideran las necesidades y la percepción de los actores implicados, se implementan herramientas de investigación cualitativa como la entrevista semiestructurada, la encuesta y la recopilación de información secundaria, para su posterior análisis. Entre los resultados, se evidencia una política municipal debilitada ya que el Estado no tiene una percepción ajustada respecto a las competencias que le corresponden según la normatividad; y se destaca de forma positiva que los tres grupos de actores coinciden en que la Comunidad es principalmente el origen del problema, donde la búsqueda de soluciones debe orientarse en esta dirección. Es de resaltar que el $88 \%$ de la Comunidad manifiesta disposición a participar y cooperar reconociendo su papel fundamental, y evidencia la necesidad de mejorar las pautas actuales, por lo cual futuros proyectos o programas con su activa participación serían viables en gran medida.

\section{PALABRAS CLAVE}

Percepción, gestión, residuos sólidos, grupos de interés.

\section{RESIDENTIALSOLIDWASTEMANAGEMENT IN VILLAVICENCIO CITY. A VIEWFROMTHESTAKEHOLDERS: COMPANY, GOVERNMENT AND COMMUNITY.}

\section{ABSTRACT}

Environmental management involves a participatory management in solving problems (García et al., 2014), about this, the article presents the results about perception of the stakeholders: Company, State and Community, about issues related to solid waste management, work focused on residential complexes in Villavicencio city, in order to understand the current situation and establish critical aspects as a starting point for improving the actual processes in terms of sustainability of solid waste management in the city. According to the methodological approach Action-Research and current regulations, that consider the needs and perceptions of stakeholders, qualitative research tools such as semi-structured interview, the survey and secondary data 
collection were implemented, for a further analysis. In the results was evidenced a weakened municipal policy since the State does not have an accurate perception of the responsibilities assigned to it by the regulations; and stands out in a positive way that the three groups of stakeholders agree that the Community is primarily the source of the problem, so the search for solutions should be oriented in this direction. Stands out that the Community expresses willingness to participate and cooperate in $88 \%$, recognizing their critical role and highlighting the need to improve current guidelines, so future projects or programs with its active participation would be largely feasible.

\section{KEY WORDS}

Perception, management, solidwaste, stakeholders.

\section{INTRODUCCIÓN}

El desarrollo local plantea que todos los actores sociales deben ser considerados elementos importantes, debido al papel social y político que desempeñacada uno desde lasinstituciones oficiales, privadas, organizaciones sociales o como ciudadano, en un determinado proceso en procura de impulsar movimientos y acciones locales (Couto, 2006; García, Paz \& Hernández, 2012). En este caso, la gestión de los residuos sólidos tiene como finalidad el bienestar y mejoramiento de la calidad de vida de la población y del ambiente, a través de un conjunto de actividades encaminadas a la reducción de residuos, aprovechamiento de los materiales, valorización energética, entre otras [Decreto 2981 (MVCT, 2013)]. Basado enqueen algunas ciudades los residuos sólidos generados con potencial de aprovechamiento son el 80\%(TaboadaGonzález,Aguilar-Virgen \& Ojeda-Benítez,2011).

De acuerdo con el enfoque metodológico Investigación-Acción, se deben considerar las necesidades de los actores según su contexto en la gestión de los residuos sólidos (Lederer, 2013). En la ciudad de Villavicencio se identifican tres grupos de actores: Empresa, Estado y Comunidad. La Empresa, representada por el sector privado, ha tenido una gran evolución en su participación en la prestación del servicio desde la entrada en vigencia de la Ley 142 de 1994 (Uribe, 2014). Por su parte el Estado,visto como los municipios, debe atender aspectos de los servicios públicos a través de funciones de gestión (Cordero, 2011). La gestión ambiental se entiende como una herramienta participativa para la resolución de problemáticas, donde se combinanherramientas administrativas, tecnológicas, económicasy jurídicas, para el mejoramiento de la calidad de vida de la población y de los recursos naturales, en un marco de sostenibilidad (Muriel, 2006; García,Toyo, Acosta, Rodríguez \& El Zauahre, 2014).

En este sentido, los principios básicos para la prestación del servicio de aseo en el marco de la Gestión Integral de Residuos Sólidos, estipulados en el Decreto 2981 de 2013, artículo 3, se destaca "garantizar la participación de los usuarios en la gestión y fiscalización de la prestación" (MVCT, 2013). Basados en esto, el objetivo de este estudio fue conocer la percepción de los grupos de interés (Empresa, Estado y Comunidad), sobre aspectos relacionados con la gestión de residuos sólidos domiciliarios en la ciudad de Villavicencio, y así contribuir con información de base que permita a los tomadores de decisiones mejorar los procesos actuales. 


\section{MATERIALES Y MÉTODOS}

\section{Área de estudio}

El presente estudio se realizó en el área urbana de la ciudad de Villavicencio, Meta, Colombia (Figura 1), ubicada a $4^{\circ} 09^{\prime} 12^{\prime \prime}$ de latitud Norte y $73^{\circ} 39^{\prime} 06^{\prime \prime}$ de longitud Oeste, con temperatura promedio anual de $25,6^{\circ} \mathrm{C}$ (Alcaldía de Villavicencio, 2013), precipitación media anual registrada de $3700 \mathrm{~mm}$, altura sobre el nivel del mar de 467 $\mathrm{m}$ y con una población estimada para el año 2014 en 473.718 habitantes (DANE, 2010).
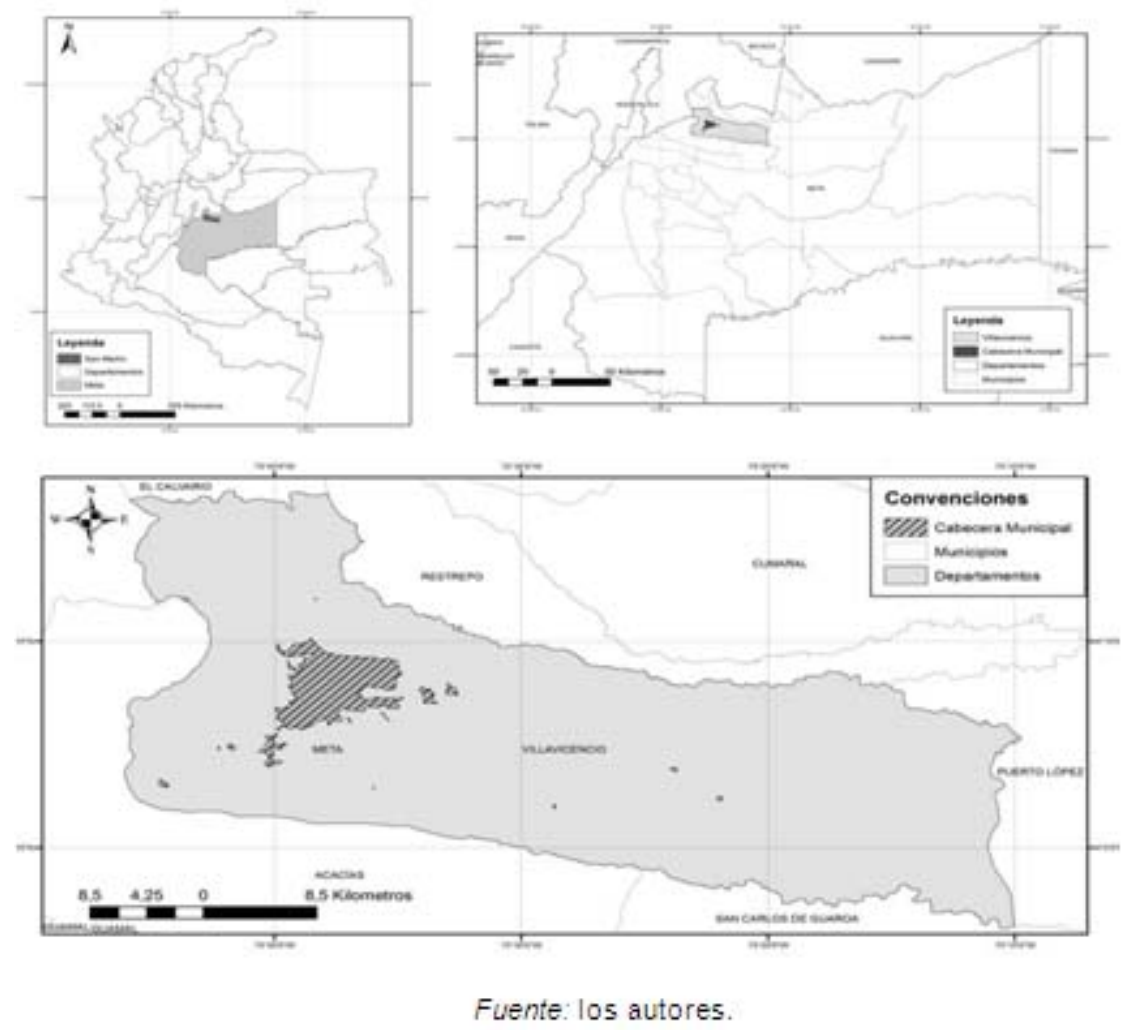

Figura 1. Localización geográfica de la ciudad de Villavicencio (Meta, Colombia).

\section{Grupos de interés o stakeholders}

Los grupos de interés o actores implicados en la gestión de residuos sólidos considerados en el estudio están al nivel de Empresa, Estado y Comunidad. En este sentido, como "Empresa" se determinó a Bioagrícola del Llano S.A. E.S.P., única prestadora del servicio público de aseo en la ciudad. El "Estado" se representa a nivel municipal por la Secretaría de Ambiente, y se incluye a la autoridad ambiental Corporación para el Desarrollo Sostenible del Área de Manejo Especial La Macarena (Cormacarena). En la "Comunidad" se delimita a los habitantes de cinco conjuntos residenciales de los estratos 3 al 5: El Triángulo, Ciudad Real, Monte Bello, Caminos de Sevilla y Montecarlo. 


\section{Recolección de información}

Las herramientas metodológicas aplicadas fueron: la entrevista semiestructurada,la encuesta y la recopilación de información secundaria (Clavijo \& Pérez, 2014). Estas herramientas de investigación cualitativa permiten profundizar a través del conocimiento del individuo y las comunidades (Chárriez, 2012). De esta manera, se recopiló la percepción de los actores, basados en que el ser humano orienta sus actos hacia las cosas en función de lo que estas significan para él (Blumer, 1982).

\section{RESULTADOS Y DISCUSIÓN}

A continuación se presentan los resultados sobre la percepción de los grupos de interés incluidos en el estudio, representados por la Empresa, el Estado y la Comunidad. En el primer apartado se contemplan tres aspectos comunes con el objetivo de presentar un punto de vista neutral exponiendo las ideas de los tres grupos de actores, y luego se dan a conocer diferentes aspectos específicos según las competencias de cada actor.

\section{Convergencia de tres puntos de vista}

\section{¿Qué es la gestión de residuos sólidos?}

Para la Empresa, la gestión de residuos sólidos corresponde a un servicio de recolección, transporte y disposición final, remitiéndose a la norma vigente. En segundo lugar, para el Estado, el secretario de Medio Ambiente habla de promover la recolección, transporte, disposición y aprovechamiento de residuos. En Cormacarena, autoridad ambiental, se relaciona el concepto con "hacer las cosas como se debe", saber qué tipo de residuos se generan, dónde se van a acopiar temporalmente, qué porcentaje va a ser aprovechado o reciclado, para finalmente llegar a la disposición final como última opción dentro de un adecuado plan de gestión.

Mientras que para la Comunidad el concepto tiene múltiples interpretaciones tales como: "proceso de recolectar, transportar, procesar, reciclar y dar un destino final adecuado a los residuos" con el $2 \%$. Otros señalan una o dos etapas de este proceso, donde "separación de residuos" se menciona en mayor proporción con el 32\%. El 20\% habla del "manejo de residuos generados". El 16\% alude a un sistema de reciclaje para evitar la contaminación. De igual forma, proporcionan diferentes definiciones en relación a los residuos sólidos con el $28 \%$. Basados en esto, para la Comunidad el concepto de gestión de residuos sólidos se concibe en general como separación en la fuente, y es de resaltar que en varios casos el término "residuos sólidos" no se entendió en primera instancia y, asimismo se hace alusión a la palabra "basura" como un sinónimo.

Moreno \& Rincón (2009) evidencian en la ciudad de Cali una tendencia a la resignificación de la basura como residuo sólido, lo que trae como consecuencia cambios en las prácticas de manejo de residuos sólidos. La percepción es un aspecto social con el cual el individuo tiende a aceptar o a rechazar objetos, eventos o situaciones. Por otro lado, para Salgado (2012) el término de residuo sólido ha empezado a sustituir el concepto de basura y los habitantes consideran que la gestión de residuos sólidos es una responsabilidad compartida. 


\section{¿Principal problemática de la gestión de residuos sólidos?}

Para la Empresa el principal problema es la cultura ciudadana, afirmación basada en su experiencia con programas voluntarios como el de "Ruta Selectiva", dirigido a empresas y conjuntos residenciales, donde han capacitado a las personas pero todavía no hay cultura de separación. Cercana a la preocupación de la Empresa, para el Estado el principal problema es la indisciplina social que involucra la comunidad, la educación al ciudadano y la indiferencia de los funcionarios públicos y del sector productivo. Para la autoridad ambiental, Cormacarena, el porcentaje de aprovechamiento debe ser mucho más alto; la empresa de aseo presta un buen servicio de rutas y horarios de recolección, pero se destaca la falta de articulación entre el usuario y la empresa, la distancia del relleno sanitario con la ciudad, y la baja cultura en la separación en la fuente.

En la Comunidad el $34 \%$ indica la falta de educación de la sociedad en cuanto al manejo y separación de residuos como el principal problema. En segunda instancia, el $18 \%$ señala la falta de concientización ante la problemática. El 14\% habla de la falta de cultura o hábitos en la sociedad y conocimiento sobre el tema. El 10\% dice que es la falta de compromiso de los habitantes, mientras que el restante $24 \%$ atribuye a diferentes aspectos de logística de la empresa de aseo para el transporte de residuos reciclables y la falta de control del municipio.

Conocer el punto de vista de la comunidad es esencial, ya que es un actor fundamental en la gestión de residuos sólidos, al ser generador y punto de partida de la problemática, ya que le compete la separación en la fuente como eslabón inicial en esta cadena (Moreno \& Rincón, 2009); se encuentra que la mayoría $(76 \%)$ es consciente de esto. Así mismo, la Empresa y el Estado coincidieron en que el problema se origina especialmente en la sociedad, teniendo en cuenta que este reside en el estilo de vida consumista de las sociedades contemporáneas (Lipovetsky, 2000). Resultado que coincide con Taboada-González et al. (2011), donde el 75\% declaró que la solución debe provenir de la organización comunitaria, fomentando actitudes de pertenencia y corresponsabilidad entre los habitantes.

\section{Forma de participación de los grupos de interés}

La Empresa destaca que es la encargada de recoger residuos sólidos domiciliarios y comerciales, y realiza una gestión valiosa para mantener la ciudad limpia con su programa de educación "Bioagrícola en tu Barrio" en cuanto al manejo de residuos y la cultura del servicio de aseo, pero existen aspectos por mejorar. El secretario de Medio Ambiente expresa que su forma de participación es realizando educación ambiental y organizando a la comunidad, colegios y sector productivo en jornadas de limpieza en caños y ríos o donde sea solicitado. Cormacarena menciona que hacen campañas con un equipo de educación ambiental en colegios, imprimen cartillas o guías ambientales y tienen un programa de recolección de pilas.

Los actores Empresa y Estado, a pesar de ser positivo su aporte en cuanto a educación como un aspecto esencial para cualquier plan de gestión de residuos sólidos, al estar condicionados "por la educación del individuo y el entorno cultural en el que interactúa" (Vásquez, 2011), en la mayoría de los casos estas iniciativas están desarticuladas. Mencionan sus gestiones actuales sin ir más allá de lo que podrían aportar, y un aspecto preocupante es encontrar una política municipal debilitada, ya que el Estado establece que no es competencia del municipio, e indica que sobre el tema se debe consultar a la Empresa de aseo, lo que muestra que no han apropiado el rol que les corresponde según la normatividad vigente. 
Finalmente, se destaca que la Comunidad tiene clara la forma en que puede aportar a mejorar los procesos actuales, ya que el $48 \%$ considera la separación en la fuente como la forma en que participaría, y enfatiza en que se debe separar en orgánicos e inorgánicos o residuos reciclables y no reciclables. En segundo lugar, el 26\% indica que participaría reciclando en el hogar. El $6 \%$ considera que podría participar al concientizar a familiares y amigos sobre esta problemática. $Y$ en una proporción del $4 \%$ destaca aspectos como reducir la generación de residuos, ser solidario comunitariamente y participar en capacitaciones.

\section{Empresa}

Bioagrícola del Llano S.A. resalta que el programa de "Ruta Selectiva", dirigido a conjuntos residenciales desde hace aproximadamente 12 años, hace una contraprestación en árboles y abono, además cuenta con un vehículo de recolección exclusivo para residuos reciclables y un total de 132 clientes, que recuperan aproximadamente $23.400 \mathrm{~kg}$ de materiales que pueden incluirse nuevamente en el ciclo de producción: 103 son empresas y colegios con $20.710 \mathrm{~kg}$ recuperados, y 29 son conjuntos residenciales con $2.690 \mathrm{~kg}$ recuperados. El programa es orientado a responsabilidad social de la empresa con el objetivo de crear cultura de los residuos sólidos, y los costos no se incluyen en la tarifa del servicio de aseo.

El esquema de "Ruta Selectiva" se realiza de la siguiente manera: inicia con la capacitación de usuarios con temas de separación en la fuente y formación de líderes. Los resultados han sido más efectivos con los clientes comerciales y el principal problema es la inadecuada separación. Dentro de las mejoras del programa está la búsqueda de estrategias para motivar a los usuarios especialmente los residenciales, ya que pasado el tiempo disminuyen el entusiasmo y el nivel de cantidad de residuos recuperados.

\section{Estado}

Respecto a la Política de Gestión de Residuos Sólidos, el secretario de Medio Ambiente indica que "no es competencia" del municipio la gestión de residuos sólidos pero trabajan limpiando los botaderos ilegales, y aclara que tienen un papel educativo, por lo cual realizan jornadas de limpieza y educación ambiental, con 1.915 toneladas de residuos recolectadas en la vía pública durante 18 meses. El municipio no tiene metas en esta materia ya que delegó a la empresa de aseo y no hay personal específico responsable de la gestión de residuos sólidos, pero -según el secretario de Medio Ambiente- existen acciones por cumplir, de acuerdo al Plan de Gestión Integral de Residuos Sólidos -PGIRS-, en cuanto a educación ambiental como un proceso de apoyo pero no como una obligación de la Secretaría.

Actualmente, los países latinoamericanos presentan tasas de urbanización altas respecto a otros lugares del mundo, allí el $80 \%$ de la población se concentra en la ciudades, este panorama es muestra de un deficiente control y un bajo nivel de planificación de las autoridades locales (ONU-Hábitat, 2012), aspectos que se evidencian claramente en el caso del municipio de Villavicencio, donde la Política de Gestión de Residuos Sólidos debe ser desarrollada ampliamente desde la Alcaldía, ya que el bajo nivel de planificación trae como consecuencia desigualdad, marginación social y grandes impactos negativos en el ambiente. La sostenibilidad urbana, es decir "el desarrollo que proporciona calidad de vida sin degradar las condiciones ambientales de la ciudad y su entorno" (Red de Ciudades Cómo Vamos, 2011), es una necesidad, y el primer paso para lograrlo es la toma de conciencia del rol y responsabilidad por parte del ente territorial. 
Por su parte, Cormacarena establece que la responsabilidad de esta gestión es del municipio, y en el PGIRS se deben plasmar las actividades a desarrollar. La corporación trabaja junto a los municipios con seguimiento y control respecto a las acciones implementadas para la gestión de residuos sólidos, otorga licencias a proyectos de relleno sanitario, verifica que no existan botaderos a cielo abierto, evalúa información de interventoría respecto a lixiviados, agua y aire, y realiza visitas de control a celdas transitorias. En el plan de acción de la Corporación Autónoma Regional -CAR- se establecen metas para cada una de las temáticas ambientales, apoyando a los municipios para organizar la gestión de residuos sólidos. Para lo cual, tienen dos funcionarios en el tema de residuos sólidos dentro del grupo Aire y Urbano, que trabaja hace tres años el tema. Es de destacar que no existe un presupuesto para la gestión de residuos sólidos.

Desde la Secretaría de Medio Ambiente se observa que el nivel de organización de los recuperadores informales es bajo, y se debe ayudar para que puedan tener una fuente de trabajo digna. Cormacarena establece que no existe un programa especial para esta población en la ciudad y no están censados. La corporación tiene una base de datos de las empresas recicladoras, a las cuales hace seguimiento. Finalmente, en cuanto al estado del proceso de actualización del PGIRS, el secretario de Medio Ambiente considera que se debe actualizar, pero se requieren estudios costosos y no hay un presupuesto para esto. Cormacarena indica que el tema lo manejan la Alcaldía y la empresa Bioagrícola del Llano S.A.

\section{Comunidad}

Respecto a las personas encuestadas en conjuntos residenciales, el $70 \%$ tiene casa propia y el $30 \%$ vive en arriendo, su tiempo de residir en el conjunto residencial es de 1 a 30 años, con un promedio de 9,4 años. El $60 \%$ femenino y el $40 \%$ masculino. El $30 \%$ se dedica al hogar, el $54 \%$ es empleado/independiente y el $16 \%$ estudiante de nivel universitario.

Respecto a la importancia de la gestión de los residuos sólidos, es fundamental encontrar que el $76 \%$ de las personas encuestadas determina como la principal razónel "minimizar el impacto ambiental", y en segundo lugar, con el $24 \%$, "la no proliferación de enfermedades". Reconocer estos aspectos es positivo en el camino para la concientización de las consecuencias negativas de la generación de los residuos sólidos en el medio ambiente, la salud y bienestar de la sociedad. Guerra (2001), en sus postulados, habla de que se deben replantear los valores y la forma de vida de una sociedad consumista, en un enfoque microético, la vida cotidiana cobra un nuevo sentido moral, para lo cual es necesario una apropiación de la conciencia ambiental, la cual determina los actos de las personas, y sería en forma definitiva y profunda al existir un entendimiento de las razones por las cuales se debe actuar de una determinada forma.

Por otro lado, el $70 \%$ de la Comunidad aplica alguna estrategia de gestión de residuos sólidos en su casa, porque sabe que acude un reciclador o recuperador que depende económicamente de estos recursos; y de este porcentaje, el $74 \%$ afirma que realiza separación en la fuente de reciclables y no reciclables, el $17 \%$ separa plástico, y el $9 \%$ manifestaron que "reciclan". En cuanto al destino de los residuos sólidos generados en los conjuntos residenciales, se encuentra que la empresa de aseo y los recicladores o recuperadores son los principales; el $2 \%$ reutiliza papel o cartón y vidrio. El $98 \%$ de los residuos de cocina tiene como destino final el relleno sanitario a través de la empresa de aseo. Finalmente, los residuos que presentan mayores niveles de recuperación son los envases PET y el papel o cartón generados en el hogar al entregarlos a un 
reciclador, con el $68 \%$ y $66 \%$ respectivamente, seguidos por las latas $(50 \%)$ y el vidrio (44\%). (Tabla 1).

Tabla 1. Destino final de los residuos sólidos generados en el hogar por tipo de residuo

\begin{tabular}{|l|c|c|c|c|}
\hline \multirow{2}{*}{ Tipo de residuo } & \multicolumn{4}{|c|}{ Destino final de los residuos sólidos } \\
\cline { 2 - 5 } & Relleno sanitario & Reutiliza & Vende & Reciclador \\
\hline Papely cartón & $32 \%$ & $2 \%$ & $0 \%$ & $66 \%$ \\
\hline Envases PET & $32 \%$ & $0 \%$ & $0 \%$ & $68 \%$ \\
\hline Latas & $50 \%$ & $0 \%$ & $0 \%$ & $50 \%$ \\
\hline Vidrio & $54 \%$ & $2 \%$ & $0 \%$ & $44 \%$ \\
\hline Residuos de cocina & $98 \%$ & $2 \%$ & $0 \%$ & $0 \%$ \\
\hline
\end{tabular}

Fuente: los autores.

Por otra parte, el $28 \%$ de las personas encuestadas manifestó que ha recibido información o capacitaciones relacionadas con la gestión de residuos sólidos en el conjunto residencial, pero no son constantes y no corresponden a un programa de capacitación. Así mismo, el $96 \%$ de la Comunidad manifiesta la necesidad de mejorar las reglas o pautas establecidas actualmente, y se destaca que el $44 \%$ realiza propuestas dirigidas a la educación como principal factor. El $17 \%$ cree que implementar la separación de residuos en los hogares es fundamental, y en igual proporción indica la importancia de establecer un punto ecológico. El 10\% considera establecer normas en cuanto a una mejor gestión de los residuos en el conjunto con el compromiso de todos. El restante $12 \%$ corresponde a propuestas como participación de la administración del conjunto residencial, la Alcaldía municipal, entre otras.

Al respecto, se encuentra que el $88 \%$ de la Comunidad está dispuesta a participar y cooperar con integrantes de su familia en programas y talleres que se emprendan en el conjunto residencial, un porcentaje positivo, ofreciendo grandes expectativas en cuanto a realizar diferentes actividades para mejorar la gestión de residuos sólidos. En este aspecto, se debe recordar que el $70 \%$ de las personas encuestadas son propietarios, por lo cual la continuidad y sostenibilidad en el tiempo de programas que se emprendan sería de largo plazo. Iniciativas que estarían justificadas ya que el $96 \%$ evidencia la necesidad de mejorar el sistema actual. Se puede llegar a pensar que en los conjuntos residenciales hay un mayor sentido de pertenencia y normas de convivencia que propenden por el bienestar de la comunidad para adelantar estos procesos, lo que contrasta con los diferentes barrios del municipio, donde se ha presentado un proceso de ciudad, en el que se alejan las personas al interior de los hogares con respecto a la vecindad (Safa, 1995). 


\section{CONCLUSIONES}

En la relación entre actores se evidencia como conflicto una baja comunicación, y como factor relevante se destaca que el Estado no tiene una percepción ajustada a la normatividad vigente respecto a las competencias que le corresponden, lo que da cuenta de una política municipal debilitada y la gran cantidad de aspectos por mejorar, aunque existe una noción acorde al concepto de gestión de residuos sólidos. La empresa de aseo, como empresa privada, tiene claridad al respecto pero no hace grandes propuestas en cuanto a su participación en el mejoramiento del sistema actual.

Los tres grupos de actores coinciden en que la Comunidad es principalmente el origen del problema, lo que es positivo en la medida en que las partes implicadas irían en la misma dirección en la búsqueda de soluciones, y es de resaltar que la Comunidad manifiesta disposición a participar y cooperar, reconociendo su papel fundamental. Acercarse a la percepción de los tres grupos de actores se constituye como un proceso sumamente importante para conocer desde dónde se pueden generar los cambios en lo cotidiano, en la concienciación de lo que es importante o no, y los conceptos que manejan, como un aspecto a considerar en el diseño de programas de educación ambiental y diferentes iniciativas a nivel municipal para el fortalecimiento de la política de gestión de residuos sólidos.

\section{AGRADECIMIENTOS}

Los autores agradecen a los habitantes de los conjuntos cerrados (El Triángulo, Ciudad Real, Monte Bello, Caminos de Sevilla y Montecarlo), por su participación en el proyecto, y a los funcionarios de las entidades públicas y privadas que aportaron sus opiniones.

\section{POTENCIAL CONFLICTO DE INTERESES}

No existe ningún conflicto de intereses respecto a la publicación de este artículo, el cual se elaboró a partir de la tesis de maestría de Ángela María Niño Torres con la asesoría de Juan Manuel Trujillo y Adriana Paola Niño Torres.

\section{REFERENCIAS}

- Alcaldía de Villavicencio. (2013). Síntesis Diagnóstica NORTE Plan de Ordenamiento Territorial Villavicencio.

- Blumer, H. (1982). El interaccionismo simbólico: perspectiva y método. Barcelona: Hora. 
- Chárriez, M. (2012). Historias de vida: Una metodología de investigación cualitativa.Revista Griot, 5(1), 50-67. ISSN 1949-4742.

- Clavijo, N., \& Pérez, M. (2014). Tubérculos andinos y conocimiento agrícola local en comunidades rurales de Ecuador y Colombia. Cuadernos de Desarrollo Rural, II (74), 149-166.

- Cordero, J.M. (2011). La administración pública y los derechos humanos en México. Ciencia y Sociedad,XXXVI, 43-162.

- Couto, S. (2006). Qué es el Desarrollo Local. Recuperado de Link

- DANE. (2010). Proyecciones demográficas por municipio basadas en el censo del año 2005. Recuperado de Link

- García, H., Toyo, L., Acosta, Y., Rodríguez, L., \&El Zauahre, M. (2014). Percepción del manejo de residuos sólidos urbanos (fracción inorgánica) en una comunidad universitaria. Multiciencias, 14 (3), 247-256.

- García, J., Paz, A., \& Hernández, P. (2012). Gestión del reciclaje de residuos sólidos desde un enfoque racional. Multiciencias, 12, 39-44.

- Guerra, M.J. (2001). Breveintroducción a la ética ecológica. Madrid: Mínimo Tránsito - Antonio Machado libros.

- Lederer, J. (2013). Actionresearchtackles. Composting in Uganda. Waste Management World Magazine. Special: Biowastefocus.

- Lipovetsky, G. (2000). El crepúsculo del deber: la ética indolora de los nuevos tiempos democráticos.Colombia: Anagrama.

- Ministerio de Vivienda, Ciudad y Territorio (MVCT). (2013). Decreto 2981. "Por el cual se reglamenta la prestación del servicio público de aseo". Diario Oficial 49010.

- Moreno, O.L., \& Rincón, M.T. (2009). Nociones de basura y prácticas en el manejo de residuos sólidos en encerramientos residenciales. Prospectiva, 14, 209-332.

- Muriel, R.D. (2006). Gestión ambiental. Idea Sostenible. Espacio de Reflexión y Comunicación en Desarrollo Sostenible, 3(13).

- ONU-Hábitat. (2012). Estado de las ciudades de América Latina y el Caribe 2012. Rumbo a una nueva transición urbana.

- Red de Ciudades Cómo Vamos. (2011). Pobreza, desigualdad social y sostenibilidad urbana: retos para las ciudades de Colombia. Boletín $N^{\circ} 5$. 
- Safa, P. (1995). El estudio de vecindarios y comunidades en las grandes ciudades.Espiral, 1(2), 113-129.

- Salgado, J.A. (2012). Residuos sólidos: percepción y factores que facilitan su separación en el hogar. El caso de estudio de dos unidades habitacionales de Tlalpan. Quivera, 14,91-112.

- Taboada-González, P.A., Aguilar-Virgen, Q., \& Ojeda-Benítez, S. (2011). Análisis estadístico de residuos sólidos domésticos en un municipio fronterizo de México.Avances en ciencias e ingeniería, 2(1), 9-20.

- Uribe, E. (2014). Reformas fiscales y regulatorias en la gestión y manejo de residuos sólidos. América Latina frente al cambio climático. Naciones Unidas CEPAL

- Vásquez, Ó.C. (2011). Gestión de los residuos sólidos municipales en la ciudad del Gran Santiago de Chile: desafíos y oportunidades. Revista internacional de contaminación ambiental, 27(4). ISSN 0188-4999.

1. Magíster en Gestión Ambiental Sostenible. Docente tiempo completo, Corporación Universitaria Minuto de Dios, Vicerrectoría Regional Llanos. Villavicencio, Colombia.aninotorres@uniminuto.edu.co ORCID 0000-0002-8967-6730

2. M.Sc. en Ciencias Ambientales. Instituto de Ciencias Ambientales de la Orinoquia Colombiana (ICAOC) - Grupo de Investigación en Gestión Ambiental Sostenible (GIGAS). Villavicencio, Colombia. jmtrujillo18@gmail.com ORCID 0000-0001-96124080

3. Administradora de Empresas. Docente catedrática, Universidad de los Llanos. Villavicencio, Colombia. adrininotorres@gmail.com ORCID 0000-0003-1388-1786

Para citar este artículo: Niño Torres, Á.M., Trujillo González, J.M., \& Niño Torres, A.P. (2017). Gestión de residuos sólidos domiciliarios en la ciudad de Villavicencio. Una mirada desde los grupos de interés: Empresa, Estado y Comunidad. Luna Azul, 44, 177-187. DOI: 10.17151/luaz.2017.44.11. Recuperado de http://200.21.104.25/lunazul/index.php/component/content/article?id=227

Esta obra está bajo una Licencia de Creative Commons Reconocimiento CC BY

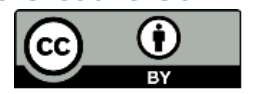

